Acta Crystallographica Section E

Structure Reports

Online

ISSN 1600-5368

\section{3-[(E)-4-Methoxybenzylidene]-1-methyl- piperidin-4-one}

\section{Gayathri, ${ }^{a}$ D. Velmurugan, ${ }^{a *}$ R. Ranjith Kumar, ${ }^{b}$ S. Perumal ${ }^{\mathrm{b}}$ and K. Ravikumar}

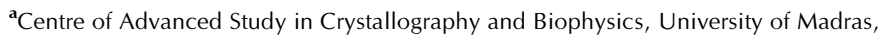
Guindy Campus, Chennai 600 025, India, 'bepartment of Organic Chemistry, School of Chemistry, Madurai Kamaraj University, Madurai 625 021, India, and 'Laboratory of X-ray Crystallography, Indian Institute of Chemical Technology, Hyderabad 500 007, India

Correspondence e-mail: d_velu@yahoo.com

Received 21 January 2008; accepted 30 January 2008

Key indicators: single-crystal X-ray study; $T=293 \mathrm{~K}$; mean $\sigma(\mathrm{C}-\mathrm{C})=0.003 \AA$; $R$ factor $=0.045 ; w R$ factor $=0.128 ;$ data-to-parameter ratio $=11.1$.

The piperidone ring of the title compound, $\mathrm{C}_{14} \mathrm{H}_{17} \mathrm{NO}_{2}$, adopts a half-chair conformation. The crystal packing is stabilized by intermolecular $\mathrm{C}-\mathrm{H} \cdots \mathrm{O}$ interactions, which generate a $C(8)$ chain running along the $b$ axis.

\section{Related literature}

For related literature, see: Abignente \& Biniecka-Picazio (1977); Angle \& Breitenbucher (1995); Cremer \& Pople (1975); Nardelli (1983); Wang \& Wuorola (1992).

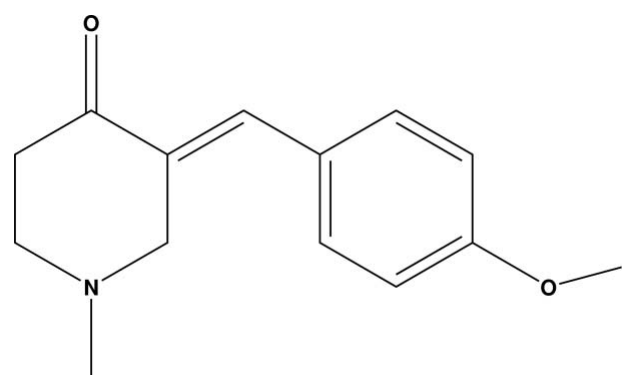

\section{Experimental}

Crystal data

$\mathrm{C}_{14} \mathrm{H}_{17} \mathrm{NO}_{2}$

$M_{r}=231.29$

Orthorhombic, $P 2_{1} 2_{1} 2_{1}$ $a=7.5212(7) \AA$

$$
\begin{aligned}
& b=12.4097(11) \AA \\
& c=13.5062(12) \AA \\
& I^{\circ}=1260.6(2) \AA^{3} \\
& Z=4
\end{aligned}
$$

Data collection

Bruker SMART CCD area-detector diffractometer

Absorption correction: none 10874 measured reflections

Refinement

$R\left[F^{2}>2 \sigma\left(F^{2}\right)\right]=0.044$

$w R\left(F^{2}\right)=0.128$

$S=1.11$

1729 reflections
Mo $K \alpha$ radiation

$\mu=0.08 \mathrm{~mm}^{-1}$

$T=293$ (2) K

$0.24 \times 0.22 \times 0.21 \mathrm{~mm}$

1729 independent reflections 1577 reflections with $I>2 \sigma(I)$ $R_{\text {int }}=0.019$

\section{Table 1}

Hydrogen-bond geometry $\left(\AA{ }^{\circ}\right)$.

\begin{tabular}{lllll}
\hline$D-\mathrm{H} \cdots A$ & $D-\mathrm{H}$ & $\mathrm{H} \cdots A$ & $D \cdots A$ & $D-\mathrm{H} \cdots A$ \\
\hline $\mathrm{C} 10-\mathrm{H} 10 \cdots \mathrm{O}^{\mathrm{i}}$ & 0.93 & 2.54 & $3.419(3)$ & 157 \\
\hline
\end{tabular}

Symmetry code: (i) $-x, y+\frac{1}{2},-z+\frac{3}{2}$.

Data collection: SMART (Bruker, 2001); cell refinement: SAINT (Bruker, 2001); data reduction: $S A I N T$; $\operatorname{program}(\mathrm{s})$ used to solve structure: SHELXS97 (Sheldrick, 2008); program(s) used to refine structure: SHELXL97 (Sheldrick, 2008); molecular graphics: PLATON (Spek, 2003); software used to prepare material for publication: SHELXL97 and PARST (Nardelli, 1995).

DG thanks the Council of Scientific and Industrial Research (CSIR), India, for a Senior Research Fellowship. Financial support from the University Grants Commission (UGC-SAP) and the Department of Science \& Technology (DST-FIST), Government of India, is acknowledged by DV for providing facilities to the department.

Supplementary data and figures for this paper are available from the IUCr electronic archives (Reference: BT2673).

\section{References}

Abignente, E. \& Biniecka-Picazio, M. (1977). Acta Pol. Pharm. 34, 241-242. Angle, S. R. \& Breitenbucher, J. G. (1995). In Studies in Natural Products Chemistry; Stereoselective Synthesis, edited by Atta-ur-Rahman, Vol. 16, Part J, pp 453-502. New York: Elsevier.

Bruker (2001). SMART and SAINT. Bruker AXS Inc., Madison, Wisconsin, USA.

Cremer, D. \& Pople, J. A. (1975). J. Am. Chem. Soc. 97, 1354-1358.

Nardelli, M. (1983). Acta Cryst. C39, 1141-1142.

Nardelli, M. (1995). J. Appl. Cryst. 28, 659.

Sheldrick, G. M. (2008). Acta Cryst. A64, 112-122.

Spek, A. L. (2003). J. Appl. Cryst. 36, 7-13.

Wang, C.-L. \& Wuorola, M. A. (1992). Org. Prep. Proced. Int. 24, 585-621. 


\section{supporting information}

Acta Cryst. (2008). E64, o552 [doi:10.1107/S1600536808003280]

\section{3-[(E)-4-Methoxybenzylidene]-1-methylpiperidin-4-one}

\section{Gayathri, D. Velmurugan, R. Ranjith Kumar, S. Perumal and K. Ravikumar}

\section{S1. Comment}

Substituted 4-piperidones are important synthetic intermediates for the preparation of various pharmaceuticals (Wang \& Wuorola, 1992). 4-Piperidones are also widely prevalent in natural products such as alkaloids (Angle \& Breitenbucher, 1995). Derivatives of 4-piperidones have been found to exhibit spasmolytic activities (Abignente \& Biniecka-Picazio, 1977). Since, the title compound is pharmacologically important, the crystal structure of the title compound has been determined by X-ray diffraction.

The sum of the bond angles around N1 $\left[331.4(6)^{\circ}\right]$ indicate the $s p^{3}$ hybridization. The torsion angles around $\mathrm{C} 10-\mathrm{C} 11$ $-\mathrm{O} 2-\mathrm{C} 14\left[179.4(2)^{\circ}\right]$ and $\mathrm{C} 12-\mathrm{C} 11-\mathrm{O} 2-\mathrm{C} 14\left[-0.8(3)^{\circ}\right]$ indicate that the methoxy group is planar with the phenyl ring.

The piperidone ring adopts a half-chair conformation with the puckering parameters (Cremer \& Pople, 1975) and the smallest displacement asymmetry parameters (Nardelli, 1983) being $\mathrm{q}_{2}=0.366$ (3) $\AA, \mathrm{q}_{3}=0.361$ (3) $\AA, \mathrm{Q}_{\mathrm{T}}=0.515$ (3) $\AA$ and $\theta=45.4(3)^{\circ}$. The molecular conformation is stabilized by weak $\mathrm{C}-\mathrm{H} \cdots \mathrm{O}$ intramolecular interactions. The crystal packing is stabilized by $\mathrm{C}-\mathrm{H} \cdots \mathrm{O}$ intermolecular interactions generating a chain $\mathrm{C}(8)$ running along $b$ axis.

\section{S2. Experimental}

A mixture of 1-methyl-4-piperidone $(1 \mathrm{mmol})$ and pyrrolidine $(1.2 \mathrm{mmol})$ was taken in a glass tube, mixed well and kept aside for $5 \mathrm{~min}$ at ambient temperature. To this mixture, 4-methoxybenzaldehyde (1 mmol) was added, mixed thoroughly and the tube containing the mixture was partially immersed in a silica bath placed in a microwave oven and irradiated at 4 power level for 8 minutes. The progress of the reaction was monitored after every $1 \mathrm{~min}$ of irradiation by TLC with petroleum ether:ethyl acetate (1:2 v/v mixture) as eluent. After each irradiation, the reaction mixture was cooled to room temperature and mixed well. The maximum temperature of the silica bath, measured immediately after each irradiation was over by stirring the silica bath with the thermometer, was found to be $65^{\circ} \mathrm{C}$. After completion of the reaction as evident from the TLC, the product was purified by column chromatography using petroleum ether:ethyl acetate $(7: 2 v / v)$ mixture and crystallized from ethyl acetate.

\section{S3. Refinement}

In the absence of anomalous scatterers Friedel pairs had been merged prior to refinement. All H-atoms were refined using a riding model with $\mathrm{d}(\mathrm{C}-\mathrm{H})=0.93 \AA, U_{\text {iso }}=1.2 U_{\text {eq }}(\mathrm{C})$ for aromatic $\mathrm{C}$ atoms, $0.97 \AA, U_{\text {iso }}=1.2 U_{\text {eq }}(\mathrm{C})$ for methylene and $0.96 \AA, U_{\text {iso }}=1.5 U_{\text {eq }}(\mathrm{C})$ for methyl groups. 


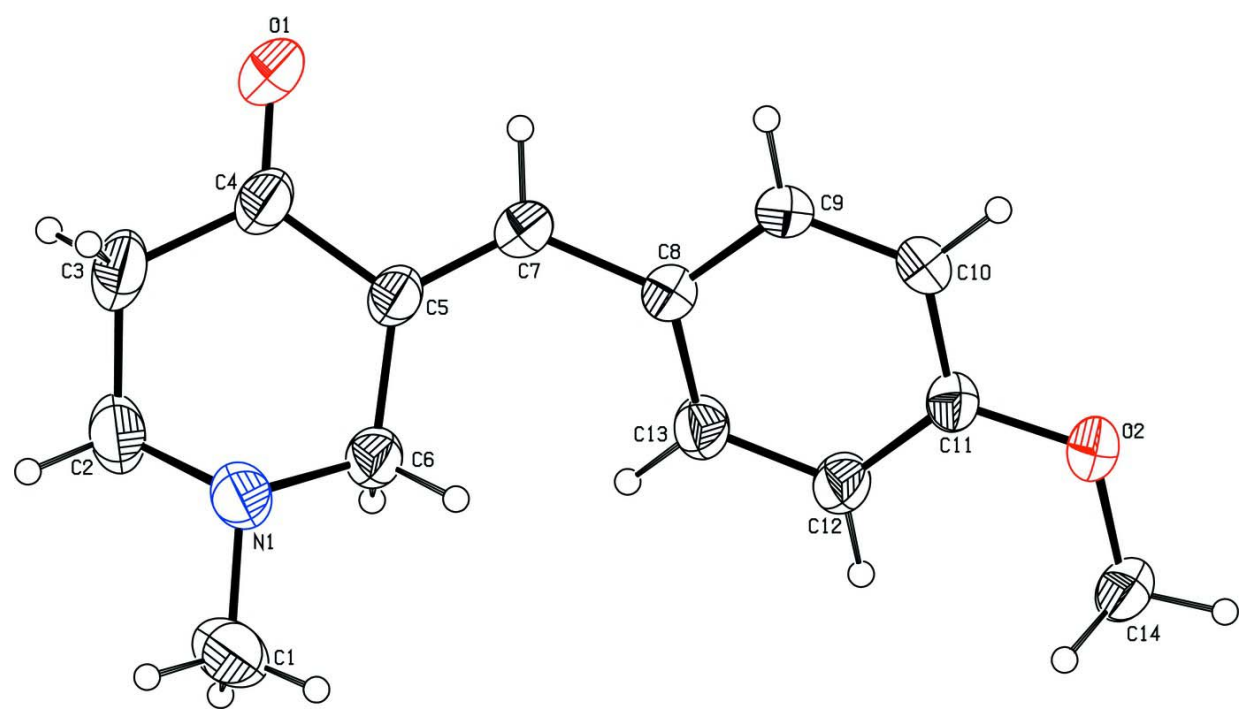

\section{Figure 1}

The molecular structure of title compound, showing 30\% probability displacement ellipsoids.

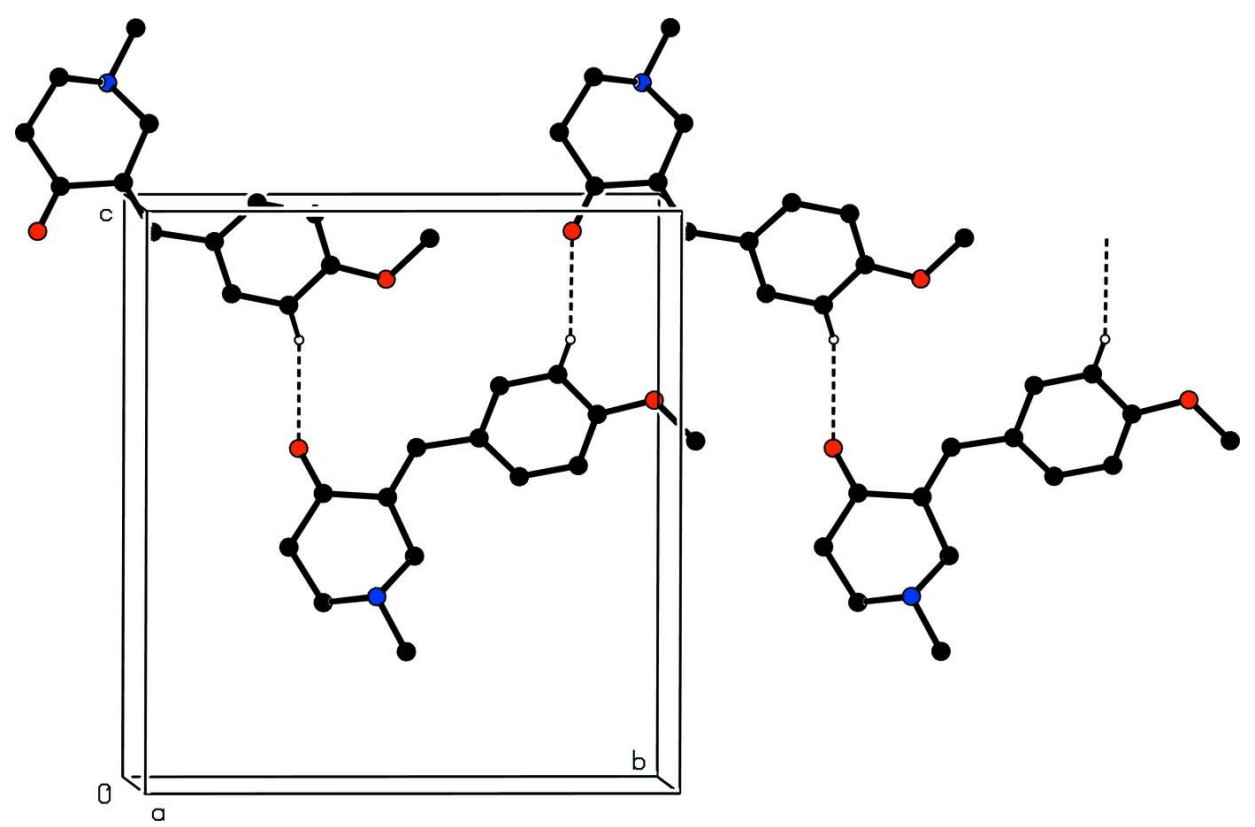

Figure 2

The molecular packing of the title compound, viewed down the $a$ axis.

\section{3-[(E)-4-Methoxybenzylidene]-1-methylpiperidin-4-one}

\section{Crystal data}

$\mathrm{C}_{14} \mathrm{H}_{17} \mathrm{NO}_{2}$

$M_{r}=231.29$

Orthorhombic, $P 22_{1} 2_{1}$

Hall symbol: P $2 \mathrm{ac} 2 \mathrm{ab}$

$a=7.5212(7) \AA$

$b=12.4097(11) \AA$

$c=13.5062(12) \AA$
$V=1260.6(2) \AA^{3}$

$Z=4$

$F(000)=496$

$D_{\mathrm{x}}=1.219 \mathrm{Mg} \mathrm{m}^{-3}$

Mo $K \alpha$ radiation, $\lambda=0.71073 \AA$

Cell parameters from 861 reflections

$\theta=2.2-25.0^{\circ}$ 
$\begin{aligned} \mu & =0.08 \mathrm{~mm}^{-1} \\ T & =293 \mathrm{~K}\end{aligned}$

\section{Data collection}

Bruker SMART CCD area-detector diffractometer

Radiation source: fine-focus sealed tube

Graphite monochromator

$\omega$ scans

10874 measured reflections

1729 independent reflections

\section{Refinement}

Refinement on $F^{2}$

Least-squares matrix: full

$R\left[F^{2}>2 \sigma\left(F^{2}\right)\right]=0.045$

$w R\left(F^{2}\right)=0.128$

$S=1.11$

1729 reflections

156 parameters

0 restraints

Primary atom site location: structure-invariant direct methods
Block, pale yellow

$0.24 \times 0.22 \times 0.21 \mathrm{~mm}$

1577 reflections with $I>2 \sigma(I)$

$R_{\text {int }}=0.019$

$\theta_{\max }=28.1^{\circ}, \theta_{\min }=2.2^{\circ}$

$h=-9 \rightarrow 9$

$k=-16 \rightarrow 16$

$l=-17 \rightarrow 17$

Secondary atom site location: difference Fourier map

Hydrogen site location: inferred from neighbouring sites

$\mathrm{H}$-atom parameters constrained

$w=1 /\left[\sigma^{2}\left(F_{\mathrm{o}}^{2}\right)+(0.083 P)^{2}+0.0611 P\right]$

where $P=\left(F_{\mathrm{o}}^{2}+2 F_{\mathrm{c}}^{2}\right) / 3$

$(\Delta / \sigma)_{\max }<0.001$

$\Delta \rho_{\max }=0.28 \mathrm{e} \AA^{-3}$

$\Delta \rho_{\min }=-0.13$ e $\AA^{-3}$

\section{Special details}

Geometry. All e.s.d.'s (except the e.s.d. in the dihedral angle between two l.s. planes) are estimated using the full covariance matrix. The cell e.s.d.'s are taken into account individually in the estimation of e.s.d.'s in distances, angles and torsion angles; correlations between e.s.d.'s in cell parameters are only used when they are defined by crystal symmetry. An approximate (isotropic) treatment of cell e.s.d.'s is used for estimating e.s.d.'s involving 1.s. planes.

Refinement. Refinement of $F^{2}$ against ALL reflections. The weighted $R$-factor $w R$ and goodness of fit $S$ are based on $F^{2}$, conventional $R$-factors $R$ are based on $F$, with $F$ set to zero for negative $F^{2}$. The threshold expression of $F^{2}>\sigma\left(F^{2}\right)$ is used only for calculating $R$-factors(gt) etc. and is not relevant to the choice of reflections for refinement. $R$-factors based on $F^{2}$ are statistically about twice as large as those based on $F$, and $R$ - factors based on ALL data will be even larger.

Fractional atomic coordinates and isotropic or equivalent isotropic displacement parameters $\left(\AA^{2}\right)$

\begin{tabular}{lllll}
\hline & $x$ & $y$ & $z$ & $U_{\text {iso }} * / U_{\text {eq }}$ \\
\hline C1 & $-0.1137(5)$ & $0.0252(2)$ & $1.28219(18)$ & $0.0864(8)$ \\
H1A & -0.0039 & 0.0433 & 1.3144 & $0.130^{*}$ \\
H1B & -0.1816 & -0.0218 & 1.3241 & $0.130^{*}$ \\
H1C & -0.1803 & 0.0898 & 1.2697 & $0.130^{*}$ \\
C2 & $0.0338(4)$ & $-0.1243(2)$ & $1.20225(17)$ & $0.0802(7)$ \\
H2A & -0.0149 & -0.1685 & 1.2549 & $0.096^{*}$ \\
H2B & 0.1533 & -0.1031 & 1.2210 & $0.096^{*}$ \\
C3 & $0.0400(5)$ & $-0.18853(18)$ & $1.1072(2)$ & $0.0845(7)$ \\
H3A & 0.1321 & -0.2429 & 1.1131 & $0.101^{*}$ \\
H3B & -0.0725 & -0.2257 & 1.0992 & $0.101^{*}$ \\
C4 & $0.0749(4)$ & $-0.12308(16)$ & $1.01586(16)$ & $0.0697(6)$ \\
C5 & $0.0494(3)$ & $-0.00418(14)$ & $1.02172(14)$ & $0.0548(5)$ \\
C6 & $0.0120(3)$ & $0.04524(15)$ & $1.12177(14)$ & $0.0558(4)$ \\
H6A & 0.1232 & 0.0685 & 1.1512 & $0.067^{*}$ \\
H6B & -0.0622 & 0.1085 & 1.1130 & $0.067^{*}$
\end{tabular}


supporting information

$\begin{array}{lllll}\text { C7 } & 0.0594(3) & 0.05043(14) & 0.93680(15) & 0.0560(5) \\ \text { H7 } & 0.0790 & 0.0090 & 0.8805 & 0.067^{*} \\ \text { C8 } & 0.0436(3) & 0.16662(14) & 0.91996(13) & 0.0510(4) \\ \text { C9 } & -0.0213(3) & 0.20203(14) & 0.82852(13) & 0.0562(5) \\ \text { H9 } & -0.0506 & 0.1515 & 0.7804 & 0.067^{*} \\ \text { C10 } & -0.0427(3) & 0.30978(15) & 0.80817(12) & 0.0576(5) \\ \text { H10 } & -0.0879 & 0.3314 & 0.7472 & 0.069^{*} \\ \text { C11 } & 0.0031(2) & 0.38597(14) & 0.87840(13) & 0.0511(4) \\ \text { C12 } & 0.0740(3) & 0.35353(14) & 0.96828(14) & 0.0585(5) \\ \text { H12 } & 0.1079 & 0.4045 & 1.0151 & 0.070^{*} \\ \text { C13 } & 0.0938(3) & 0.24434(17) & 0.98781(15) & 0.0581(5) \\ \text { H13 } & 0.1422 & 0.2229 & 1.0481 & 0.070^{*} \\ \text { C14 } & 0.0194(4) & 0.57070(15) & 0.92477(17) & 0.0667(6) \\ \text { H14A } & -0.0449 & 0.5570 & 0.9849 & 0.100^{*} \\ \text { H14B } & -0.0116 & 0.6407 & 0.8999 & 0.100^{*} \\ \text { H14C } & 0.1448 & 0.5681 & 0.9379 & 0.100^{*} \\ \text { N1 } & -0.0762(3) & -0.02883(15) & 1.18885(13) & 0.0656(5) \\ \text { O1 } & 0.1230(4) & -0.16737(12) & 0.94008(14) & 0.0993(7) \\ \text { O2 } & -0.0251(2) & 0.49113(10) & 0.85323(10) & 0.0627(4)\end{array}$

Atomic displacement parameters $\left(\AA^{2}\right)$

\begin{tabular}{lllllll}
\hline & $U^{11}$ & $U^{22}$ & $U^{33}$ & $U^{12}$ & $U^{13}$ & $U^{23}$ \\
\hline C1 & $0.105(2)$ & $0.0972(18)$ & $0.0569(12)$ & $-0.0171(16)$ & $0.0036(13)$ & $0.0011(12)$ \\
C2 & $0.1040(18)$ & $0.0634(11)$ & $0.0731(13)$ & $-0.0072(14)$ & $-0.0128(14)$ & $0.0222(11)$ \\
C3 & $0.1134(19)$ & $0.0459(9)$ & $0.0942(16)$ & $-0.0027(13)$ & $-0.0080(17)$ & $0.0165(11)$ \\
C4 & $0.0965(15)$ & $0.0404(8)$ & $0.0723(12)$ & $0.0031(10)$ & $-0.0171(13)$ & $-0.0023(9)$ \\
C5 & $0.0662(11)$ & $0.0391(7)$ & $0.0590(10)$ & $0.0011(8)$ & $-0.0092(9)$ & $-0.0002(7)$ \\
C6 & $0.0660(11)$ & $0.0464(8)$ & $0.0551(9)$ & $-0.0030(8)$ & $-0.0093(9)$ & $0.0009(7)$ \\
C7 & $0.0701(11)$ & $0.0430(8)$ & $0.0551(9)$ & $0.0031(8)$ & $-0.0019(9)$ & $-0.0053(7)$ \\
C8 & $0.0603(10)$ & $0.0433(8)$ & $0.0493(8)$ & $0.0005(8)$ & $0.0021(8)$ & $0.0001(6)$ \\
C9 & $0.0787(12)$ & $0.0476(8)$ & $0.0423(7)$ & $-0.0009(9)$ & $0.0058(9)$ & $-0.0040(6)$ \\
C10 & $0.0793(12)$ & $0.0538(9)$ & $0.0397(7)$ & $0.0015(10)$ & $0.0022(9)$ & $0.0058(7)$ \\
C11 & $0.0602(9)$ & $0.0421(7)$ & $0.0510(8)$ & $-0.0017(8)$ & $0.0055(8)$ & $0.0055(7)$ \\
C12 & $0.0744(11)$ & $0.0443(9)$ & $0.0568(10)$ & $-0.0085(9)$ & $-0.0103(9)$ & $-0.0004(7)$ \\
C13 & $0.0713(11)$ & $0.0488(9)$ & $0.0544(9)$ & $-0.0029(9)$ & $-0.0150(9)$ & $0.0057(7)$ \\
C14 & $0.0878(14)$ & $0.0414(8)$ & $0.0708(12)$ & $-0.0001(10)$ & $0.0100(12)$ & $-0.0005(8)$ \\
N1 & $0.0784(11)$ & $0.0618(9)$ & $0.0565(8)$ & $-0.0120(9)$ & $-0.0084(9)$ & $0.0061(7)$ \\
O1 & $0.169(2)$ & $0.0465(7)$ & $0.0828(11)$ & $0.0139(12)$ & $-0.0067(13)$ & $-0.0083(8)$ \\
O2 & $0.0866(10)$ & $0.0415(6)$ & $0.0600(7)$ & $-0.0016(7)$ & $-0.0008(8)$ & $0.0077(5)$ \\
& & & & & & \\
\hline
\end{tabular}

Geometric parameters $\left(\AA,{ }^{\circ}\right)$

\begin{tabular}{llll}
\hline $\mathrm{C} 1-\mathrm{N} 1$ & $1.456(3)$ & $\mathrm{C} 7-\mathrm{C} 8$ & $1.465(2)$ \\
$\mathrm{C} 1-\mathrm{H} 1 \mathrm{~A}$ & 0.9600 & $\mathrm{C} 7-\mathrm{H} 7$ & 0.9300 \\
$\mathrm{C} 1-\mathrm{H} 1 \mathrm{~B}$ & 0.9600 & $\mathrm{C} 8-\mathrm{C} 13$ & $1.383(3)$ \\
$\mathrm{C} 1-\mathrm{H} 1 \mathrm{C}$ & 0.9600 & $\mathrm{C} 8-\mathrm{C} 9$ & $1.399(3)$ \\
$\mathrm{C} 2-\mathrm{N} 1$ & $1.457(3)$ & $\mathrm{C} 9-\mathrm{C} 10$ & $1.374(3)$
\end{tabular}




\begin{tabular}{|c|c|c|c|}
\hline $\mathrm{C} 2-\mathrm{C} 3$ & $1.511(4)$ & C9-H9 & 0.9300 \\
\hline $\mathrm{C} 2-\mathrm{H} 2 \mathrm{~A}$ & 0.9700 & $\mathrm{C} 10-\mathrm{C} 11$ & $1.383(3)$ \\
\hline $\mathrm{C} 2-\mathrm{H} 2 \mathrm{~B}$ & 0.9700 & $\mathrm{C} 10-\mathrm{H} 10$ & 0.9300 \\
\hline $\mathrm{C} 3-\mathrm{C} 4$ & $1.500(3)$ & $\mathrm{C} 11-\mathrm{O} 2$ & $1.365(2)$ \\
\hline $\mathrm{C} 3-\mathrm{H} 3 \mathrm{~A}$ & 0.9700 & $\mathrm{C} 11-\mathrm{C} 12$ & $1.385(3)$ \\
\hline C $3-\mathrm{H} 3 \mathrm{~B}$ & 0.9700 & $\mathrm{C} 12-\mathrm{C} 13$ & $1.389(3)$ \\
\hline $\mathrm{C} 4-\mathrm{O} 1$ & $1.217(3)$ & $\mathrm{C} 12-\mathrm{H} 12$ & 0.9300 \\
\hline $\mathrm{C} 4-\mathrm{C} 5$ & $1.490(3)$ & $\mathrm{C} 13-\mathrm{H} 13$ & 0.9300 \\
\hline $\mathrm{C} 5-\mathrm{C} 7$ & $1.334(3)$ & $\mathrm{C} 14-\mathrm{O} 2$ & $1.421(3)$ \\
\hline $\mathrm{C} 5-\mathrm{C} 6$ & $1.510(3)$ & $\mathrm{C} 14-\mathrm{H} 14 \mathrm{~A}$ & 0.9600 \\
\hline $\mathrm{C} 6-\mathrm{N} 1$ & $1.451(3)$ & $\mathrm{C} 14-\mathrm{H} 14 \mathrm{~B}$ & 0.9600 \\
\hline C6-H6A & 0.9700 & $\mathrm{C} 14-\mathrm{H} 14 \mathrm{C}$ & 0.9600 \\
\hline C6-H6B & 0.9700 & & \\
\hline $\mathrm{N} 1-\mathrm{C} 1-\mathrm{H} 1 \mathrm{~A}$ & 109.5 & $\mathrm{C} 5-\mathrm{C} 7-\mathrm{H} 7$ & 115.5 \\
\hline $\mathrm{N} 1-\mathrm{C} 1-\mathrm{H} 1 \mathrm{~B}$ & 109.5 & $\mathrm{C} 8-\mathrm{C} 7-\mathrm{H} 7$ & 115.5 \\
\hline $\mathrm{H} 1 \mathrm{~A}-\mathrm{C} 1-\mathrm{H} 1 \mathrm{~B}$ & 109.5 & $\mathrm{C} 13-\mathrm{C} 8-\mathrm{C} 9$ & $117.47(16)$ \\
\hline $\mathrm{N} 1-\mathrm{C} 1-\mathrm{H} 1 \mathrm{C}$ & 109.5 & $\mathrm{C} 13-\mathrm{C} 8-\mathrm{C} 7$ & $124.17(18)$ \\
\hline $\mathrm{H} 1 \mathrm{~A}-\mathrm{C} 1-\mathrm{H} 1 \mathrm{C}$ & 109.5 & $\mathrm{C} 9-\mathrm{C} 8-\mathrm{C} 7$ & $118.33(16)$ \\
\hline $\mathrm{H} 1 \mathrm{~B}-\mathrm{C} 1-\mathrm{H} 1 \mathrm{C}$ & 109.5 & $\mathrm{C} 10-\mathrm{C} 9-\mathrm{C} 8$ & $121.53(17)$ \\
\hline $\mathrm{N} 1-\mathrm{C} 2-\mathrm{C} 3$ & $109.93(19)$ & $\mathrm{C} 10-\mathrm{C} 9-\mathrm{H} 9$ & 119.2 \\
\hline $\mathrm{N} 1-\mathrm{C} 2-\mathrm{H} 2 \mathrm{~A}$ & 109.7 & $\mathrm{C} 8-\mathrm{C} 9-\mathrm{H} 9$ & 119.2 \\
\hline $\mathrm{C} 3-\mathrm{C} 2-\mathrm{H} 2 \mathrm{~A}$ & 109.7 & $\mathrm{C} 9-\mathrm{C} 10-\mathrm{C} 11$ & $119.92(17)$ \\
\hline $\mathrm{N} 1-\mathrm{C} 2-\mathrm{H} 2 \mathrm{~B}$ & 109.7 & $\mathrm{C} 9-\mathrm{C} 10-\mathrm{H} 10$ & 120.0 \\
\hline $\mathrm{C} 3-\mathrm{C} 2-\mathrm{H} 2 \mathrm{~B}$ & 109.7 & $\mathrm{C} 11-\mathrm{C} 10-\mathrm{H} 10$ & 120.0 \\
\hline $\mathrm{H} 2 \mathrm{~A}-\mathrm{C} 2-\mathrm{H} 2 \mathrm{~B}$ & 108.2 & $\mathrm{O} 2-\mathrm{C} 11-\mathrm{C} 12$ & $123.75(16)$ \\
\hline $\mathrm{C} 4-\mathrm{C} 3-\mathrm{C} 2$ & $114.74(18)$ & $\mathrm{O} 2-\mathrm{C} 11-\mathrm{C} 10$ & $116.37(16)$ \\
\hline $\mathrm{C} 4-\mathrm{C} 3-\mathrm{H} 3 \mathrm{~A}$ & 108.6 & $\mathrm{C} 12-\mathrm{C} 11-\mathrm{C} 10$ & $119.88(16)$ \\
\hline $\mathrm{C} 2-\mathrm{C} 3-\mathrm{H} 3 \mathrm{~A}$ & 108.6 & $\mathrm{C} 11-\mathrm{C} 12-\mathrm{C} 13$ & $119.43(17)$ \\
\hline $\mathrm{C} 4-\mathrm{C} 3-\mathrm{H} 3 \mathrm{~B}$ & 108.6 & $\mathrm{C} 11-\mathrm{C} 12-\mathrm{H} 12$ & 120.3 \\
\hline $\mathrm{C} 2-\mathrm{C} 3-\mathrm{H} 3 \mathrm{~B}$ & 108.6 & $\mathrm{C} 13-\mathrm{C} 12-\mathrm{H} 12$ & 120.3 \\
\hline $\mathrm{H} 3 \mathrm{~A}-\mathrm{C} 3-\mathrm{H} 3 \mathrm{~B}$ & 107.6 & $\mathrm{C} 8-\mathrm{C} 13-\mathrm{C} 12$ & $121.69(18)$ \\
\hline $\mathrm{O} 1-\mathrm{C} 4-\mathrm{C} 5$ & $122.0(2)$ & $\mathrm{C} 8-\mathrm{C} 13-\mathrm{H} 13$ & 119.2 \\
\hline $\mathrm{O} 1-\mathrm{C} 4-\mathrm{C} 3$ & $119.95(19)$ & $\mathrm{C} 12-\mathrm{C} 13-\mathrm{H} 13$ & 119.2 \\
\hline $\mathrm{C} 5-\mathrm{C} 4-\mathrm{C} 3$ & $118.0(2)$ & $\mathrm{O} 2-\mathrm{C} 14-\mathrm{H} 14 \mathrm{~A}$ & 109.5 \\
\hline $\mathrm{C} 7-\mathrm{C} 5-\mathrm{C} 4$ & $116.75(17)$ & $\mathrm{O} 2-\mathrm{C} 14-\mathrm{H} 14 \mathrm{~B}$ & 109.5 \\
\hline $\mathrm{C} 7-\mathrm{C} 5-\mathrm{C} 6$ & $124.98(16)$ & $\mathrm{H} 14 \mathrm{~A}-\mathrm{C} 14-\mathrm{H} 14 \mathrm{~B}$ & 109.5 \\
\hline $\mathrm{C} 4-\mathrm{C} 5-\mathrm{C} 6$ & $118.27(16)$ & $\mathrm{O} 2-\mathrm{C} 14-\mathrm{H} 14 \mathrm{C}$ & 109.5 \\
\hline $\mathrm{N} 1-\mathrm{C} 6-\mathrm{C} 5$ & $112.74(16)$ & $\mathrm{H} 14 \mathrm{~A}-\mathrm{C} 14-\mathrm{H} 14 \mathrm{C}$ & 109.5 \\
\hline $\mathrm{N} 1-\mathrm{C} 6-\mathrm{H} 6 \mathrm{~A}$ & 109.0 & $\mathrm{H} 14 \mathrm{~B}-\mathrm{C} 14-\mathrm{H} 14 \mathrm{C}$ & 109.5 \\
\hline $\mathrm{C} 5-\mathrm{C} 6-\mathrm{H} 6 \mathrm{~A}$ & 109.0 & $\mathrm{C} 6-\mathrm{N} 1-\mathrm{C} 1$ & $109.72(18)$ \\
\hline $\mathrm{N} 1-\mathrm{C} 6-\mathrm{H} 6 \mathrm{~B}$ & 109.0 & $\mathrm{C} 6-\mathrm{N} 1-\mathrm{C} 2$ & $109.5(2)$ \\
\hline $\mathrm{C} 5-\mathrm{C} 6-\mathrm{H} 6 \mathrm{~B}$ & 109.0 & $\mathrm{C} 1-\mathrm{N} 1-\mathrm{C} 2$ & $112.18(19)$ \\
\hline $\mathrm{H} 6 \mathrm{~A}-\mathrm{C} 6-\mathrm{H} 6 \mathrm{~B}$ & 107.8 & $\mathrm{C} 11-\mathrm{O} 2-\mathrm{C} 14$ & $117.27(15)$ \\
\hline $\mathrm{C} 5-\mathrm{C} 7-\mathrm{C} 8$ & $128.98(17)$ & & \\
\hline $\mathrm{N} 1-\mathrm{C} 2-\mathrm{C} 3-\mathrm{C} 4$ & $46.7(3)$ & $\mathrm{C} 8-\mathrm{C} 9-\mathrm{C} 10-\mathrm{C} 11$ & $1.1(3)$ \\
\hline $\mathrm{C} 2-\mathrm{C} 3-\mathrm{C} 4-\mathrm{O} 1$ & $163.0(3)$ & $\mathrm{C} 9-\mathrm{C} 10-\mathrm{C} 11-\mathrm{O} 2$ & $-178.8(2)$ \\
\hline
\end{tabular}




\section{supporting information}

$\begin{array}{llll}\mathrm{C} 2-\mathrm{C} 3-\mathrm{C} 4-\mathrm{C} 5 & -16.8(4) & \mathrm{C} 9-\mathrm{C} 10-\mathrm{C} 11-\mathrm{C} 12 & 1.3(3) \\ \mathrm{O} 1-\mathrm{C} 4-\mathrm{C} 5-\mathrm{C} 7 & 8.4(4) & \mathrm{O} 2-\mathrm{C} 11-\mathrm{C} 12-\mathrm{C} 13 & 178.5(2) \\ \mathrm{C} 3-\mathrm{C} 4-\mathrm{C} 5-\mathrm{C} 7 & -171.8(2) & \mathrm{C} 10-\mathrm{C} 11-\mathrm{C} 12-\mathrm{C} 13 & -1.7(3) \\ \mathrm{O} 1-\mathrm{C} 4-\mathrm{C} 5-\mathrm{C} 6 & -172.5(3) & \mathrm{C} 9-\mathrm{C} 8-\mathrm{C} 13-\mathrm{C} 12 & 2.7(3) \\ \mathrm{C} 3-\mathrm{C} 4-\mathrm{C} 5-\mathrm{C} 6 & 7.3(3) & \mathrm{C} 7-\mathrm{C} 8-\mathrm{C} 13-\mathrm{C} 12 & -179.2(2) \\ \mathrm{C} 7-\mathrm{C} 5-\mathrm{C} 6-\mathrm{N} 1 & 151.3(2) & \mathrm{C} 11-\mathrm{C} 12-\mathrm{C} 13-\mathrm{C} 8 & -0.4(3) \\ \mathrm{C} 4-\mathrm{C} 5-\mathrm{C} 6-\mathrm{N} 1 & -27.8(3) & \mathrm{C} 5-\mathrm{C} 6-\mathrm{N} 1-\mathrm{C} 1 & -178.0(2) \\ \mathrm{C} 4-\mathrm{C} 5-\mathrm{C} 7-\mathrm{C} 8 & -178.6(2) & \mathrm{C} 5-\mathrm{C} 6-\mathrm{N} 1-\mathrm{C} 2 & 58.5(2) \\ \mathrm{C} 6-\mathrm{C} 5-\mathrm{C} 7-\mathrm{C} 8 & 2.3(4) & \mathrm{C} 3-\mathrm{C} 2-\mathrm{N} 1-\mathrm{C} 6 & -68.5(3) \\ \mathrm{C} 5-\mathrm{C} 7-\mathrm{C} 8-\mathrm{C} 13 & 30.6(4) & \mathrm{C} 3-\mathrm{C} 2-\mathrm{N} 1-\mathrm{C} 1 & 169.4(2) \\ \mathrm{C} 5-\mathrm{C} 7-\mathrm{C} 8-\mathrm{C} 9 & -151.3(2) & \mathrm{C} 12-\mathrm{C} 11-\mathrm{O} 2-\mathrm{C} 14 & -0.8(3) \\ \mathrm{C} 13-\mathrm{C} 8-\mathrm{C} 9-\mathrm{C} 10 & -3.1(3) & \mathrm{C} 10-\mathrm{C} 11-\mathrm{O} 2-\mathrm{C} 14 & 179.37(19) \\ \mathrm{C} 7-\mathrm{C} 8-\mathrm{C} 9-\mathrm{C} 10 & 178.8(2) & & \end{array}$

Hydrogen-bond geometry $\left(A,{ }^{\circ}\right)$

\begin{tabular}{lllll}
\hline$D-\mathrm{H} \cdots A$ & $D-\mathrm{H}$ & $\mathrm{H} \cdots A$ & $D \cdots A$ & $D-\mathrm{H} \cdots A$ \\
\hline $\mathrm{C} 10-\mathrm{H} 10 \cdots \mathrm{O}^{\mathrm{i}}$ & 0.93 & 2.54 & $3.419(3)$ & 157 \\
\hline
\end{tabular}

Symmetry code: (i) $-x, y+1 / 2,-z+3 / 2$. 